\title{
Comparative Evaluation of the Dentin Binding Strength of Zirconium-based Polymers, Conventional Glass-ionized Hydromorphone, and Resin-modified Glass-ionized Hydromorphone
}

\author{
Kishor Sapkale, Rucha Sane, Sayed Abrar Bashir Ahmed
}

\begin{abstract}
Background: Bond strength of Glass Ionomer cements with tooth structure directly influence the clinical performance of the glass ionomer cement. In this in vitro study, aims to measure and compare micro-tensile bond strength (ptbs) of Zirconomer- a newly introduced Glass Ionomer Cement with Conventional Glass Ionomer Cement and Resin-modified Glass Ionomer cement (RMGIC). Materials and methods: The specimens were divided into 3 groups: 1) Group I: Comprises of 4 teeth in which dentin was bonded to conventional GIC, 2) Group II: Comprises of 4 teeth in which dentin was bonded to RMGIC, 3) Group III: Comprises of 4 teeth in which dentin was bonded to zirconomer. After GIC build-ups,specimens were sectioned vertically into $2 x 2 \times 6$-mm3 beams and each specimen was subjected toptbstest. Results: Intergroup comparison using One-way ANOVA followed by pairwise comparison using post hoc test showed that $\mu$ tbsof RMGIC was significantly higher than both Conventional GIC and Zirconomer (p<0.05.) Mtbs values for zirconomer were higher than those for Conventional GIC however the difference was not statistically significant.
\end{abstract}

Keywords: Conventional glass ionomer; dentin; micro-tensile bond strength; resin- modified glass ionomer cement; zirconomer

\section{Introduction}

Restoration of tooth structure lost due to dental caries using a biomimetic material is the mainstay of current dental practice. The search for a restorative material resembling the tooth structure in terms of physicomechanical, biological and aesthetic aspects continues.

The general dissatisfaction with the clinical performance of silicate cement and the need for a durable cement adhering to tooth structure directed the research towards the development of glass ionomer cement.

In 1972, Wilson and Kent introduced glass ionomer cement to the field of dentistry and the material has found multiple applications in clinical dentistry ever since. Glass ionomer cement possesses a number of clinically desirable properties such as fluoride release and uptake, chemical bonding with tooth structure, good biocompatibility and similar coefficient of thermal expansion as that of dentin. ${ }^{1}$

However, their use in clinical dentistry as a direct restorative material in high stress- bearing areas is restricted because of relatively poor mechanical properties, such as low fracture strength, toughness and wear resistance along with moisture sensitivity when compared with other direct restorative materials such as amalgam and composites. Due to these limitations along with wide chemical diversity of its structural components glass ionomer cement possess a great potential for further development. ${ }^{2}$

Through the years 1972 to the late 1980 multiple modifications were suggested such as water hardening glass ionomer cement, cermet, metal- reinforced glass ionomer cement and dispersed phase glass containing glass ionomers.

Despite all these modifications, two of the problems remained unaddressed- moisture sensitivity and lack of command cure. In an attempt to improve the mechanical properties of conventional gics, Resin-Modified Glass Ionomer Cement was developed. Resin modified Glass Ionomer Cement retained the most desirable qualities of conventional versions, namely fluoride release and ion exchange adhesion to conditioned dentin, and had a few additional advantages namely improved mechanical properties and low interfacial shrinkage stress, reduced moisture sensitivity. ${ }^{3}$ However, theses improvement in mechanical properties still could not promise its use in stress- bearing areas as a direct restorative material. Since mechanical properties of a restorative material directly influence their clinical performance, several strategies have been suggested to enhance the mechanical properties, such as the addition of zirconia, hydroxyapatite, fluorapatite, etc. Among these, zirconia modified glass ionomer cement or zirconomer is a well- known method for enhancing the mechanical properties of GIC. ${ }^{4}$

A high strength restorative material, which has been reinforced with zirconia filler particles known as zirconomer, has been a recent substitute to GIC in dentistry. ${ }^{1}$

Zirconomer also known as 'white amalgam' defines a new class of glass ionomer that supposedly promises more strength and durability than conventional GIC by the manufacturers but has not been studied in detail yet. Bond strength of a glass ionomer cement with dentin is an extremely important parameter for the assessment of the strength of adhesion between restorative material and dentin. Thus, this in vitro study was designed to evaluate and compare the microtensile bond strength of three glass ionomer cements- Conventional Glass Ionomer Cement, Resin Modified Glass Ionomer Cement, and Zirconomer.

As bonding techniques and materials have improved, the bond strengths have become high enough to cause cohesive failures during testing. ${ }^{5}$ Hence for this study micro-tensile bond testing method was used which made it possible to evaluate the bond strength with dentin more accurately. 
This study aims to evaluate and compare the microtensile bond strength of three glass ionomer cements- Conventional Glass Ionomer Cement, Resin Modified Glass Ionomer Cement, and Zirconomer.

\section{Materials and Methods}

This study involved three groups:

1) Group I: Comprises of 4 teeth in which dentin was bonded to conventional GIC

2) Group II: Comprises of 4 teeth in which dentin was bonded to RMGIC

3) Group III: Comprises of 4 teeth in which dentin was bonded to zirconomer

Each group consisted of four teeth; 6 specimens were prepared from each tooth. Each group had 24 specimens.

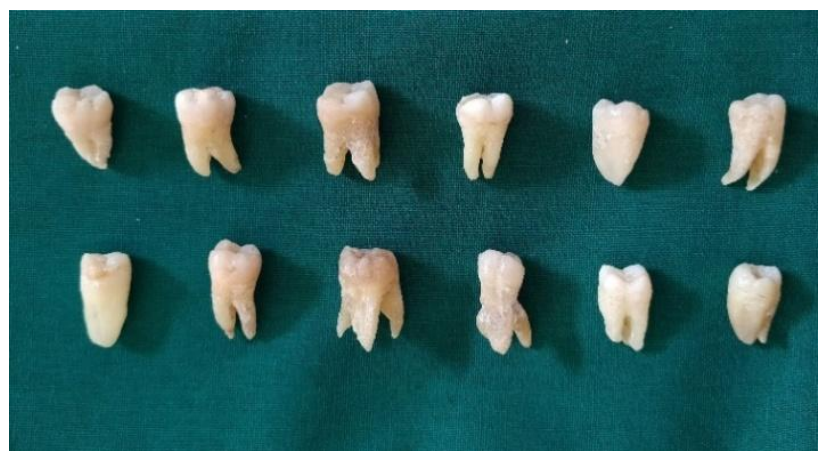

Figure 1: Recently extracted human molars

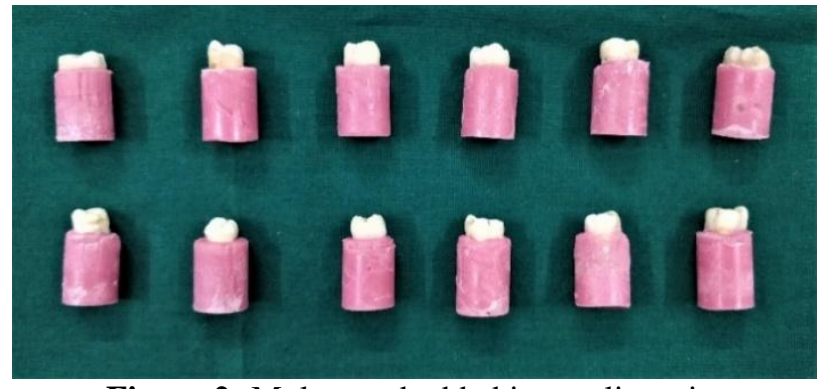

Figure 2: Molars embedded in acrylic resin

\subsection{Materials}

For this study, GC gold label high strength posterior restorative glass ionomer (GC gold label 2GI, GC Corporation, Tokyo, Japan), GC gold label LC universal restorative glass ionomer (GC gold label 2 LC, GC Corporation, Tokyo, Japan) and Zirconomer ( Zirconomer improved- Zirconia reinforced glass ionomer cement, Shofu, Japan) was used.

The sample size was determined using the mean and standard deviation values from literature to be 24 samples per group. 12 freshly extracted human molars with sound crowns and roots were obtained. Teeth with open apices, resorptive defects, and fracture lines were excluded.

After the extraction, periodontal tissues were removed and the teeth were placed in formalin until further required. Teeth were embedded in acrylic resin and the occlusal enamel was removed perpendicular to their long axis to expose a flat, midcoronal dentin surface using a low speed, diamond disc. This surface was polished with silicon carbide paper and then rinsed under copious running water to produce the smear layer and immediately was dried with moisture- free air. 12 Teeth were randomly divided into three groups.

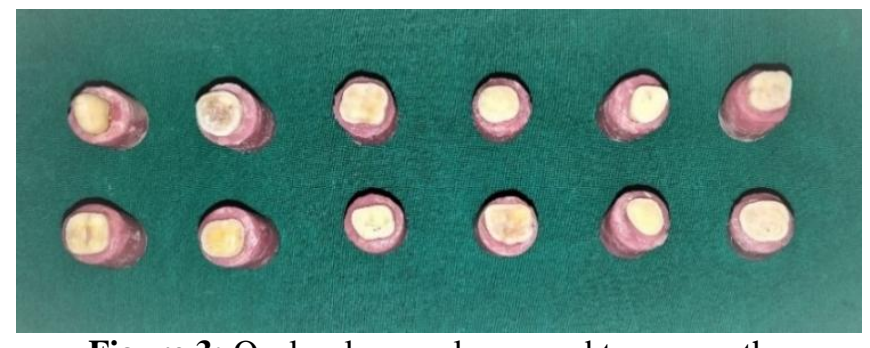

Figure 3: Occlusal enamel removed to expose the midcoronal dentin

Procedures for the application of Glass ionomer cements-

Group I- Conventional GIC was mixed according to the manufacturer's instructions. It was built up over the exposed dentinal surface in an incremental fashion until the thickness of $4 \mathrm{~mm}$ was achieved. For this build up, a stain-less steel matrix band along with the universal retainer was used. The markings were made on the matrix band to obtain the thickness of $4 \mathrm{~mm}$.
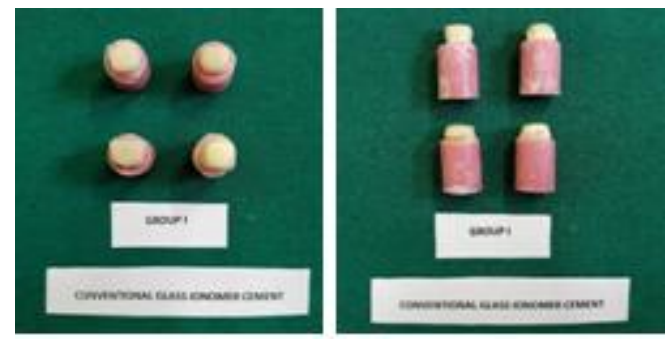

Figure 4: Conventional GIC bonded to dentin

Group II- RMGIC was mixed according to the manufacturer's instructions. It was built up over the exposed dentinal surface in incremental fashion.

Each increment was light cured for $30 \mathrm{sec}$. The procedure was repeated until the thickness of $4 \mathrm{~mm}$ was achieved. For this build up, a stain-less steel matrix band along with the universal retainer was used. The markings were made on the matrix band to obtain the thickness of $4 \mathrm{~mm}$.
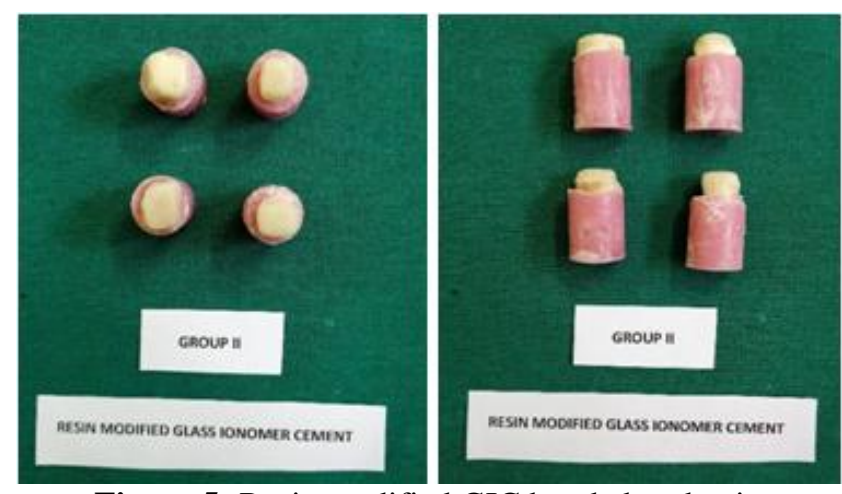

Figure 5: Resin modified GIC bonded to dentin 
Group III- Zirconomer was mixed according to the manufacturer's instructions. It was built up over the exposed dentinal surface in incremental fashion until the thickness of $4 \mathrm{~mm}$ was achieved. For this build up, a stain-less steel matrix band along with the universal retainer was used. The markings were made on the matrix band to obtain the thickness of $4 \mathrm{~mm}$.
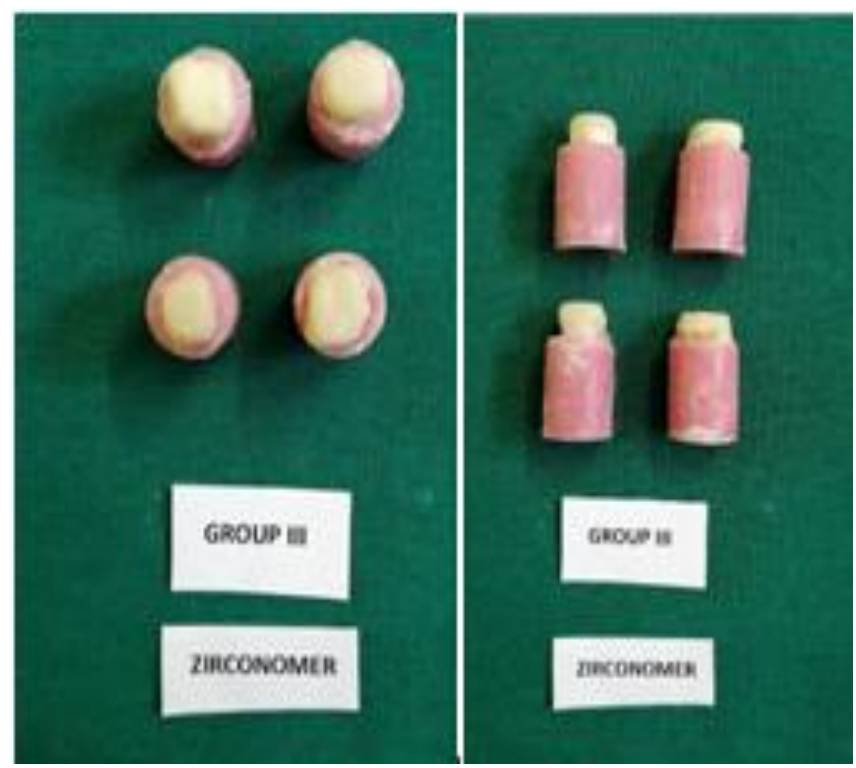

Figure 6: Zirconomer bonded to dentin

Each tooth was sectioned to obtain beams of specific dimensions. Sectioning was started in the mesio distal direction, then the tooth was rotated at $90^{\circ}$ and sectioned in the bucco lingual direction to obtain beams with an approximate cross-sectional area of $4 \mathrm{~mm}^{2}$. Length of each specimens was $6 \mathrm{~mm}$.

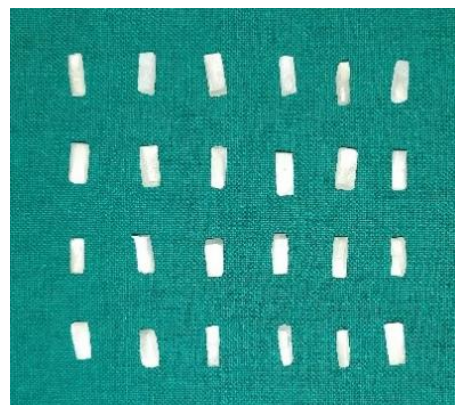

Figure 7: Specimens for Group I

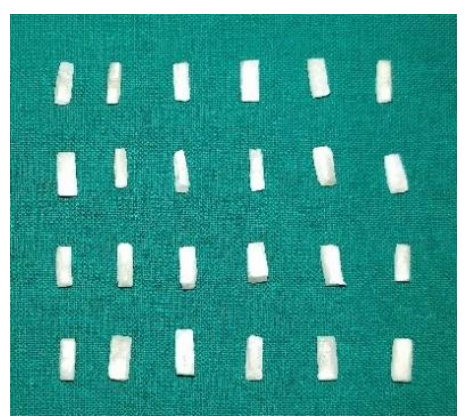

Figure 8: Specimens for Group II

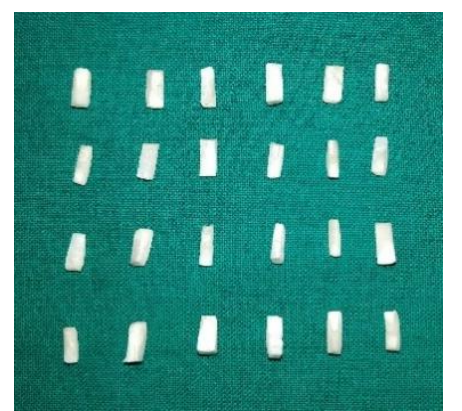

Figure 9: Specimens for Group III

\section{Micro-tensile bond strength test-}

From every group, 24 such beams were selected randomly. These were mounted to a custom acrylic jig with the help of cyanoacrylate and subjected to micro tensile bond strength testing in a Universal Testing Machine (computerized software based-ACME, India. Model No. UNITEST 10, Accuracy of the machine: $\pm 1 \%$ ) with the cross-head speed of $1 \mathrm{~mm} /$ minute.

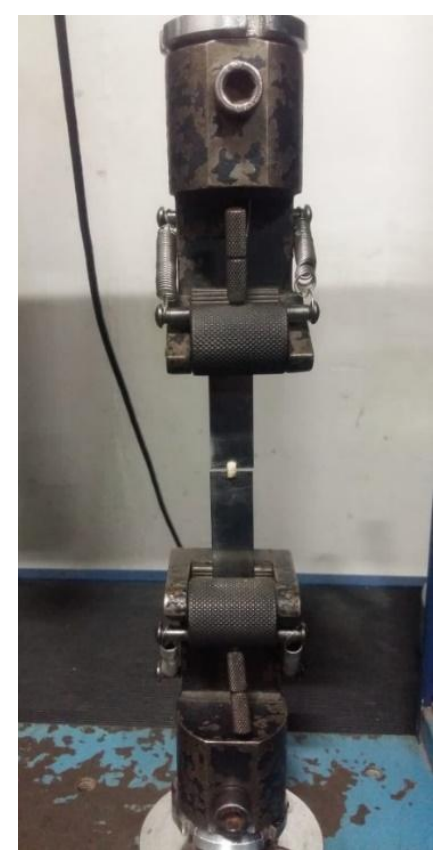

Figure 10: Universal Testing Machine (computerized software based-ACME, India. Model No. UNITEST 10., Accuracy of the machine: $\pm 1 \%$ ) with the cross-head speed of $1 \mathrm{~mm} /$ minute

Maximum load required to de-bond the specimen was measured for each specimen from every group (N) and an average value was calculated for every group. These values were used to calculate the micro-tensile bond strength by dividing the applied load by cross- sectional area of the specimen in mpaor $\mathrm{N} / \mathrm{mm} 2$. Also, the average value was calculated for all the three groups.

\section{Statistical Analysis}

Data obtained was compiled on a MS Office Excel Sheet (v 2010, Microsoft Redmond Campus, Redmond, Washington, United States).

Normality of numerical data was checked using ShapiroWilk test \& was found that the data followed a normal 
curve; hence parametric tests have been used for comparisons. Intergroup comparison ( $>2$ groups) was done using one-way ANOVA followed by pairwise comparison using post hoc test.

For all the statistical tests, $\mathrm{p}<0.05$ was considered to be statistically significant, keeping $\alpha$ error at $5 \%$ and $\beta$ error at $20 \%$, thus giving power to the study as $80 \%$.

\section{Results}

The average values of Maximum load (N) needed to debond the specimens and Micro-tensile bond strength (mpa) for all the three groups are presented in Table 1. Intergroup comparison has been demonstrated in Table 2 and Figure 11.

Table 1: Average values of Maximum load $(\mathrm{N})$ needed to de-bond the specimens and Micro-tensile bond strength

(mpa) for all the three groups

\begin{tabular}{|c|c|c|}
\hline & \multicolumn{2}{|c|}{ Average values } \\
\hline & Maximum Load (N) & Micro-tensile bond strength \\
\hline Group I & 36.16 & 2.15 \\
\hline Group II & 27.29 & 2.97 \\
\hline Group III & 29.96 & 2.20 \\
\hline
\end{tabular}

Table 2: Intergroup comparison of Micro Tensile Bond Strength (mpa)

\begin{tabular}{|c|c|c|c|c|c|c|c|c|}
\hline & \multicolumn{9}{|c|}{} & \multicolumn{2}{c|}{ 95\% Confidence Interval for Mean } & & \\
\hline & $\mathrm{N}$ & Mean & Std. Deviation & Std. Error & Lower Bound & Upper Bound & Minimum & Maximum \\
\hline 1 & 24 & 2.155833 & 0.4021347 & 0.0820854 & 1.986027 & 2.32564 & 1.34 & 2.72 \\
\hline 2 & 24 & 2.97625 & 0.5798711 & 0.1183657 & 2.731392 & 3.221108 & 1.9 & 3.8 \\
\hline 3 & 24 & 2.282083 & 0.3531717 & 0.0720909 & 2.132952 & 2.431215 & 1.81 & 3.12 \\
\hline Total & 72 & 2.471389 & 0.5776234 & 0.0680736 & 2.335654 & 2.607124 & 1.34 & 3.8 \\
\hline
\end{tabular}

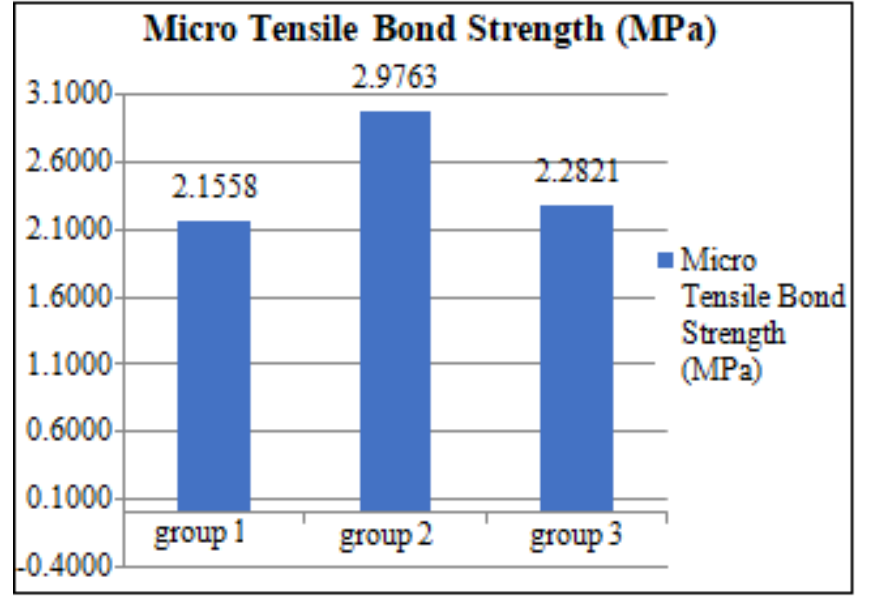

Figure 11: Graphical representation of Intergroup comparison demonstrating Micro-tensile bond strengths

The highest Mean Micro-tensile bond strength was observed in Resin- modified glass ionomer cement and dentin (Group II) followed by Zirconomer (Group III) and Conventional GIC (Group I).
One-way ANOVA showed that there was a statistically significant / highly significant difference seen for the values between the groups $(p<0.01,0.05)$ with the highest value in group 2 and least value in group 1 as demonstrated in Table 3.

Table 3: ANOVA test

\begin{tabular}{|c|c|c|c|c|c|}
\hline & Sum of Squares & Df & Mean Square & $\mathrm{F}$ & P value \\
\hline Between Groups & 9.367 & 2 & 4.684 & 22.564 & $.000^{* * *}$ \\
\hline Within Groups & 14.322 & 69 & 0.208 & & \\
\hline Total & 23.689 & 71 & & & \\
\hline \multicolumn{6}{|c|}{$\begin{array}{l}*=\text { statistically significant difference }(\mathrm{p}<0.05) \\
* *=\text { statistically highly significant difference }(\mathrm{p}<0.01) \\
\#=\text { non significant difference }(\mathrm{p}>0.05)\end{array}$} \\
\hline
\end{tabular}

For the pairwise comparison among the groups, Turkey's Post Hoc Test was used. (Table 4) Which showed a statistically significant / highly significant difference i.e. $\mathrm{P}<0.01,0.05$ for the values between the pair Group I vs group II and the pair Group II vs Group III

But there was a statistically non- significant difference seen for the values between group I vs group III $(\mathrm{p}>0.05)$.

Table 4: Pairwise comparison using Tukey's Post Hoc Tests

\begin{tabular}{|c|c|c|c|c|c|c|}
\hline \multirow{2}{*}{ (I) groups } & \multirow{2}{*}{$(\mathrm{J})$ groups } & \multicolumn{5}{|c|}{$95 \%$ Confidence Interval } \\
\cline { 3 - 7 } & & Mean Difference (I-J) & Std. Error & Sig. & Lower Bound & Upper Bound \\
\hline 1 & 2 & $-.8204167^{*}$ & 0.1315182 & $.000^{* *}$ & -1.135444 & -0.50539 \\
\hline 1 & 3 & -0.12625 & 0.1315182 & $.604 \#$ & -0.441277 & 0.188777 \\
\hline 2 & 3 & $.6941667^{*}$ & 0.1315182 & $.000^{* *}$ & 0.37914 & 1.009194 \\
\hline
\end{tabular}

\section{Discussion}

Glass ionomer cements have gained a prominent place in modern restorative dentistry. The clinical success of this restorative material largely depends upon good adhesion with tooth surface in order to resist various dislodging forces acting within the oral cavity. ${ }^{6}$ Thus, evaluation of the strength of adhesion between the tooth and restorative material holds great clinical significance. Over the years, clinicians have relied upon various laboratory tests to assess the clinical performance of a newly introduced restorative material. The validity of these tests is largely influenced by appropriate selection of the test method. ${ }^{7}$

Hence this study was designed to comparatively evaluate micro-tensile bond strength of conventional glass ionomer cement, resin- modified glass ionomer cement and zirconomer. 
This study includes micro-tensile strength test for the evaluation of bond strength. Compared to conventional tensile and shear bond strength tests, this method has several advantages. It allows measurement of adhesion in small areas, reduction in the number of teeth samples required and reduces the chance of cohesive failures occurring. ${ }^{8}$

In the present study, the mean value of micro-tensile bond strength was found to be highest for Resin modified GIC, followed by zirconomer, and was lowest for conventional GIC.

Conventional glass ionomer cement was introduced with the intention of developing a material that would adhere to the tooth surface and would have desirable aesthetic and biological properties. However, several drawbacks of this material such as moisture sensitivity and lack of command cure, led to the development of Resin modified glass ionomer cement. Intergroup comparison showed that the micro-tensile bond strength value for RMGIC was significantly higher than that for conventional GIC. These results are in agreement with the results of a study conducted by Poggio et al. ${ }^{9}$ This study reported a higher shear bond of RMGIC to dentin than GIC (Fuji IX GP Extra) when effects of dentin surface treatments on shear bond strength of gicswere evaluated. Similar results were observed by D. T. Millet who compared the bond strengths of RMGIC, modified composite and conventional GIC with tooth structure for bonding of orthodontic brackets. ${ }^{10}$

Conventional GIC is a product of an acid-base reaction between basic fluoroaluminosilicate glass powder and polycarboxylic acid. Its mechanism of bonding is based on bond formation between carboxyl groups of polyacrylic acid with hydroxyapatite at the tooth surface. The lowest microtensile bond strength was observed for this group. It could be because they are susceptible to attack by moisture during the initial setting period.

The better performance of Resin modified GIC is thought to be due to their expected dual mechanism of adhesion or enhanced mechanical properties. The adhesion is probably through a combination of a dynamic ion exchange process and micromechanical bonding mechanism. ${ }^{11}$

However, improvement in mechanical properties still cannot promise its use in stress- bearing areas as a direct restorative material. Since mechanical properties of a restorative material directly influence their clinical performance, several strategies have been suggested to enhance the mechanical properties, such as the addition of zirconia. This led to the development of zirconomer- zirconia modified glass ionomer cement. Zirconia (zro2) is a white crystalline oxide of zirconium. It is a polycrystalline ceramic that exists in several forms. The name zirconium comes from the Arabic word 'zargon' which means golden in colour.

The manufacturers claim that zirconomer possesses mechanical properties similar to those of dental amalgam while it also retains the desirable properties of GIC such as fluoride release and chemical adhesion. However, this material has not been studied in detail yet. Studies evaluating microleakage, hardness, compressive strength of zirconomer are available in the literature. ${ }^{12}$ However, there exists a void in literature regarding studies evaluating bond strength of zirconomer to dentin. In this study, micro-tensile bond strength for resin- modified glass ionomer cement was found to be higher than that for ziconomer and the difference was statistically significant. It could be because even though addition of zirconia filler particles improves the mechanical properties of zirconomer, it however, does not improve upon the issue of moisture sensitivity and early bond failure. Mechanism of bonding of zirconomer with the dentin is chemical in nature, thus lacks the reinforcement of bond with micromechanical interlocking. Micro- tensile bond strength of zirconomer was found to be higher than that of conventional glass ionomer cement, however, the difference is not statistically significant. Thus, in general terms, it is found to be comparable with the micro- tensile bond strength of conventional glass ionomer cement. This study was designed in an effort to contribute the information to the literature regarding micro-tensile bond strength of zirconomer to dentin. However, this study design suffers from a few drawbacks. This study does not take into consideration the effect of teeth sectioning on resultant bond strength also, this being an in vitro evaluation of bond strength of zirconomer the results cannot be generalised to all clinical scenarios. Thus, further in vitro studies are needed to gather sufficient evidence regarding the bond strength of zirconomer to dentin.

\section{Conclusion}

The mean value of micro-tensile bond strength was found to be the highest for RMGIC, followed by zirconomer and was the least for conventional GIC. However, it is difficult to comment upon the influence of these results on clinical situations as literature lacks sufficient evidence regarding this. This study attempts to evaluate the clinical performance of a novel restorative material- zirconomer in terms of its adhesion to dentin. However, further studies are necessary to fully understand the performance of zirconomer in an oral environment.

\section{Financial Support and Sponsorship}

\section{Conflicts of interest}

There are no conflicts of interest.

\section{References}

[1] Choi K, Oshida Y, Platt JA, Cochran MA, Matis BA, Yi $\mathrm{K}$. Microtensile bond strength of glass ionomer cements to artificially created carious dentin. Oper Dent2006;31:590-97.

[2] Prosser HJ, Powis DR, Brant P and Wilson AD. The characterization of glass ionomer cements.J Dent 1984;12:231-40.

[3] Hewlett ER, Mount GJ. Glass ionomers in contemporary restorative dentistry - a clinical update. J Calif Dent Assoc 2003;31(6):483-92. 
[4] Moshaverinia, A.; Ansari, S.; Movasaghi, Z.; Billington, R.W.; Darr, J.A.; Rehman, I.U. Modification of conventional glass-ionomer cements with Nvinylpyrrolidone containing polyacids, nano-hydroxy and fluoroapatite to improve mechanical properties. Dent Mater 2008;24:1381-90.

[5] Pashley DH, Carvalho RM, Sano H, Nakajima M, Yoshiyama M, Shono Y et al. The microtensile bond test: Areview. J Adhes Dent 1999;1(4):299-309.

[6] Manuja N, Pandit IK, Srivastava N, Gugnani N, Nagpal R. Comparative evaluation of shear bond strength of various esthetic restorative materials to dentin: an in vitro study. J Indian Soc PedoPrev Dent 2011;29(1):713.

[7] Van Meerbeek B, De Munck J, Yoshida Y, Inoue S, Vargas M, Vijay P, et al. Buonocore memorial lecture. Adhesion to enamel and dentin: current status and future challenges. Oper Dent 2003;28:215-35.

[8] Pashley DH, Sano H, Ciucchi B, Yoshiyama M, Carvalho RM. Adhesion testing of dentin bonding agents: a review. Dent Mater1995;11(2):117-25.

[9] Poggio C, Beltrami R, Scribante A, Colombo M, Lombardini M. Effects of dentin surface treatments on shear bond strength of glass-ionomer cements. Ann Stomatol (Roma) 2014 janmar;5(1):15-22.

[10] Millett DT, mccabe JF. Orthodontic bonding with glass ionomer cement: a review. Eur J Orthod1996;18:38599.

[11] Prabhakar AR, Raj S, Raju OS. Comparison of shear bond strength of composite, compomer and resin modified glass ionomer in primary and permanent teeth. An in vitro study. J Indian Soc PedoPrev Dent2003;21(3):86-94.

[12] Abdulsamee N, Elkhadem AH. Zirconomer and zirconomer improved (white amalgams): Restorative materials for the future. Review. EC Dent Sci 2017; $15: 134-50$. 